\title{
Point Charge Has A Details
}

\author{
Kareem Hassaan \\ Unaffiliated
}

9 February 2022

\begin{abstract}
I Talk About The Electric Field Due to point Charge does not exist in a certain short distance interval Hence I talk about This forbidden distance That Electric Field doesn't exist within it Also I Talk about threshold point That Electric Field Start in Existence and take a value at It
\end{abstract}

\section{Contents}

1 Introduction 1

2 There Is a Solid Angle 3

3 Electric Field Singularity 5

4 Forbidden Distance $\quad 7$

5 Forbidden Area $\quad 7$

6 Threshold Value of Distance $\quad 8$

7 Results and Discussion $\quad 8$

\section{Introduction}

In Coulomb Torsion Balance Experiment Charles Coulomb Say His Famous Law Coulomb's Law[1] and If We see This Experiment Apparatus We Find Two Spheres and every Sphere is Charged and Has A total Charge Q1 , Q2 respectively. 
The Electrostatic Force Between Two Charged Sphere Is

$F(r)=\frac{1}{4 \pi \varepsilon_{0}} \frac{Q 1 Q 2}{r^{2}}$

Q1: Total Charge on First Sphere

Q2: Total Charge on Second Sphere

$\varepsilon_{0}$ : Permittivity of Free Space

r : Separation Distance Between Two Spheres

Eq.1. Only Depend On $\mathrm{r}$ as a independent Variable

Also We Could Use Eq. 1. To Calculate a Force Between Two Point Charges q1 , q2 Respectively Because Eq.1. does not Depend on spheres Radii

Note : Point Charge is A Charged Point Without any Features.

The Electrostatic Force Eq.1. Could Be Written as

$F=Q 1 E$

F : Electrostatic Force

Q1: Total Charge on First Sphere

E: Electric Field Due To Second Charged Sphere

The Force Per Unit Charge Called An Electric Field

$$
E=\frac{F}{Q 1}
$$

The Electric Field Equation is

$E(r)=\frac{1}{4 \pi \varepsilon_{0}} \frac{Q 2}{r^{2}}$ 


\section{There Is a Solid Angle}

If we Put a Point Charge at The Origin of Any Coordinate System in Euclidean Space This Point Charge Has an Total Charge Q

If We Use a Gauss's Law [3] To Calculate The Total Charge Of Point Charge We Have a Gaussian Surface and This Gaussian Surface Is Closed Spherical Surface S With Volume V enclosed This Point Charge

The Integral Form Of Gauss's Law Could Be Expressed As

$$
\phi_{E}=\frac{Q}{\varepsilon_{0}}
$$

Since

Q is Total Charge Enclosed By Gaussian Surface

$\varepsilon_{0}$ Is Permittivity of Free Space

$$
\phi_{E}=\oiint_{s} E . d A
$$

Where $\phi_{E}$ is The Electric Flux Through a Closed Gaussian Surface S enclosing any volume V, Q is The Total charge enclosed By Surface S and located in V , The Electric Flux $\phi_{E}$ Is Defined as Double Integral Of The Electric Field Eq.6.

We Could Use Any Coordinate System To Determine Position in Euclidean Space, For Example We Will Use Spherical Coordinate System $(\mathrm{r}, \theta, \phi)$

Area Element in Spherical Coordinate System Equal to

$\mathrm{dA}=\mathrm{r}^{2} \operatorname{Sin}(\theta) d \theta d \phi$

Gauss's Law Say 


$$
\phi_{E}=\oiint_{s} E . d A=\frac{Q}{\varepsilon_{0}}
$$

I will Take a Closed Spherical Surface S as a Gaussian Surface If We Substitute By Eq.4. and Eq.7. in Eq.8. and Take The Left Hand Side in Eq.8. we Will Obtain This Form .

Let

$$
\left(\frac{Q}{4 \pi \varepsilon_{0}}\right)=C
$$

$\mathrm{C} \iint_{S} \frac{\hat{r}}{r^{2}} \cdot \hat{n} d A$

Since

$\mathrm{I}=\iint_{S} \frac{\hat{r}}{r^{2}} \cdot \hat{n} d A$

C I This Is the Left Hand Side of Eq.8. and I Will Take Only The Part I

$$
\begin{gathered}
I=\int_{0}^{2 \pi} d \phi \int_{0}^{\pi} \frac{1}{r^{2}} r^{2} \operatorname{Sin} \theta d \theta \\
I=4 \pi \\
C=\frac{Q}{4 \pi \varepsilon_{0}} \\
C I=\frac{Q}{4 \pi \varepsilon_{0}} 4 \pi
\end{gathered}
$$

in Eq.9. Which Mentioned in Denominator is Came From Coulomb Constant 
$\mathrm{K}_{E}=\frac{1}{4 \pi \epsilon_{0}}$

in Eq.9. $4 \pi$ Which Mentioned in Numerator is Came Form Solid Angle Of Total Sphere

$\Omega=\frac{A}{r^{2}}$

So

$\Omega=4 \pi$

From Our Previous Statements We Know $4 \pi$ in Coulomb Constant Eq.10. is Just a Solid Angle of Total Sphere Eq.11.

Consequently an Electric Field Due to Single Point Charge Which Calculated From Coulomb Torsion Balance Experiment it is Equation Contain An Angular Part Equal To Solid angle Has a Value $4 \pi$ As We See in Eq.4.

So By Using Eq.11. We Could Write Eq.4. As A Following

$$
\begin{aligned}
E(r) & =\frac{1}{\Omega \epsilon_{0}} \frac{Q^{2}}{r^{2}} \\
E(A) & =\frac{Q^{2}}{A \epsilon_{0}}
\end{aligned}
$$

\section{A : Area Of a Gaussian Closed Spherical Surface}

Therefore Electric Field After This Substitution Depend on Area as a Dependent Variable ( Which Take Symbol A ) Instead Of Radial Distance $r$ as an Independent Variable

\section{$3 \quad$ Electric Field Singularity}

Electric Field due to A Point charge Located at origin only depend explicitly on The Magnitude of This Position radius Vector ( Which Denoted By r )

In Electric Field Equation was Mentioned in Eq.4. We Have only one Singularity [2] equal to $0($ singularity $=0)$ 
If we Want to Calculate Electric Field at $r=0$ we Find $E(r)$ is undefined and Does Not Exist Which Means this Value of $r(r=0)$ is Singularity

$\mathrm{r}$ is a magnitude of position radius Vector and This Magnitude only Takes positive Values r \& 0 Because Point Charge Located At Origin

This Is a Plotting Of Only Radial Part Of E(r) Function, Then I Will Have a Graph as a Following

Radial Part is $\frac{1}{r^{2}}$

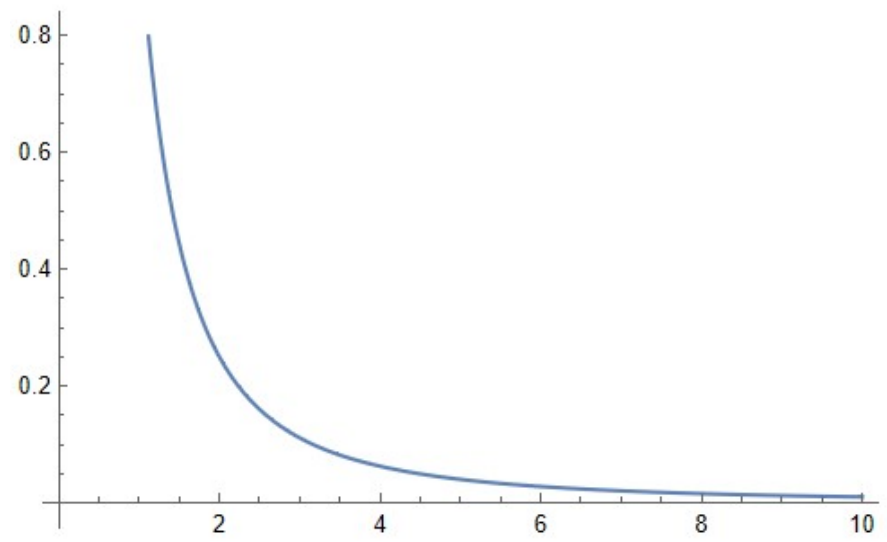

Figure 1: Figure 1: Plot of Radial Part in Electric Field Due to Point Charge $\frac{1}{r^{2}}$ on The Vertical and $\mathrm{r}$ on The Horizontal, Plot Domain from 0 to 10 i.e. $(0,10]$ Semi Open Interval .

Also We Find a problem in a Generalized domain of $r$ i.e. Generalized Semi Open Interval $0<r \leq b$

If We Take a limit from positive direction Side

$$
\begin{aligned}
& \lim _{x \rightarrow 0^{+}} E(r)=\lim _{x \rightarrow 0^{+}} \frac{1}{4 \pi \varepsilon_{0}} \frac{Q}{r^{2}} \\
& \lim _{x \rightarrow 0^{+}} E(r)=\frac{Q}{4 \pi \varepsilon_{0}} \lim _{x \rightarrow 0^{+}} \frac{1}{r^{2}} \\
& \frac{Q}{4 \pi \varepsilon_{0}} \lim _{x \rightarrow 0^{+}} \frac{1}{r^{2}}=\infty^{+}(\text {Limit Does Not Exist })
\end{aligned}
$$


This Means That Limit Does Not Exist .

\title{
4 Forbidden Distance
}

\author{
$(\varepsilon, \delta) \quad$ Definition Of Limit [4] States That
}

For every real $\varepsilon>0$, there exists a real $\delta>0$ such that for all real $\mathrm{x}$, $0<|x-p|<\delta$ implies that $0<|f(x)-L|<\varepsilon$

Then If I Reverse The Previous Statement and Apply it in our Previous Case ( Eq.11. ) We will See That The Magnitude of This Radial Vector $r$ Cannot Take Any Value in This Closed Interval $[0, \delta]$ Consequently The Electric Field

Is Undefined and Does Not Exist in This interval $[0, \delta]$, So We Could Say This Interval is Forbidden Distance.

Forbidden Distance $=[0, \delta]$

\section{Forbidden Area}

If I Use a Formula of Electric Field Which Depend on Area As an Rather Than Radial Distance Eq.12

By Using Equations Eq.11 and Eq.12. and Our Pervious Conclusion I Find a Forbidden Area Instead Of Forbidden Radial Distance In This Case

$$
A=4 \pi r^{2}
$$

Eq. 14 is Area of Sphere With Radius r

By Using Eq.14. The Forbidden Area of Gaussian Closed Spherical Surface S Equal to $4 \pi \delta^{2}$ 
The Forbidden Area Equal to

$A_{\text {Forbidden }}=4 \pi \delta^{2}$

$\delta$ : is Forbidden Radial Distance ( Forbidden Distance)

\section{Threshold Value of Distance}

The Electric Field Still Does Not Exist Until The Value Of Radial Distance r Become Bigger Than Forbidden Distance $\delta$

So

$E(\delta)=\frac{1}{4 \pi \varepsilon_{0}} \frac{Q}{\delta^{2}}$

$E(\delta)=\infty^{+}$

Electric Field Does Not Exist at This point $\delta$ and Start at Next Positive points of $\mathrm{r}$.

Since $r>\delta$

\section{$7 \quad$ Results and Discussion}

Every Point Charge Has an Electric Field and This Field Does Not Exist at distance equal to zero and this point is Just a Singularity $(r=0)$ Moreover Every singularity Has a Characteristic Domain Closed interval equal to $[0, \delta]$ and Electric Field also Does Not Exist in this Characteristic Interval and The Electric Field Start at Value r Since $r>\delta>0$

So Electric Field

$$
E(r)=\text { does not exist } \quad \text { at } \quad[0, \delta]
$$



$E(r)=e x i s t$
at
$r>\delta$

\section{References}

[1] Isobel Falconer. Charles augustin coulomb and the fundamental law of electrostatics. Metrologia, 41(5):S107, 2004.

[2] Erwin Kreyszig. Advanced Engineering Mathematics 9th Edition with Wiley Plus Set. John Wiley \& Sons, 2007.

[3] Chandralekha Singh. Student understanding of symmetry and gauss's law of electricity. American journal of physics, 74(10):923-936, 2006.

[4] James Stewart. Calculus. Cengage Learning, 2011. 